\title{
NORMAL RELATIVE INTEGRAL BASES FOR QUARTIC FIELDS OVER QUADRATIC SUBFIELDS
}

\author{
Blair K. Spearman and Kenneth S. Williams*
}

\begin{abstract}
Let $L$ be a quartic number field with a quadratic subfield $K$. In 1986 Kawamoto gave a necessary and sufficient condition for $L$ to have a normal relative integral basis (NRIB) over $K$. In this paper the authors explicitly construct a NRIB for $L / K$ when such exists using their previous work on relative integral bases. The special cases when $L$ is bicyclic, cyclic and pure are examined in detail.
\end{abstract}

\section{Introduction}

Let $L$ be a quartic number field with quadratic subfield $K=Q(\sqrt{c})$, where $Q$ denotes the rational number field. Then $L=Q(\sqrt{c}, \sqrt{a+b \sqrt{c}})$, where $a+b \sqrt{c}$ is not a square in $Q(\sqrt{c})$, and where $a, b$ and $c$ may be taken to be integers with both $c$ and the greatest common divisor $(a, b)$ squarefree. Let $O_{L}$ (resp. $O_{K}$ ) denote the ring of integers of $L$ (resp. $K$ ). In this paper we assume that $L$ has a relative integral basis (RIB) over $K$, and determine when $L$ has a normal relative integral basis (NRIB) over $K$. Those $L$ which have a relative integral basis (RIB) over $K$ have been characterized in [9]. It is shown in [9, Theorem 2] that such $L$ have a RIB over $K$ of the form $\{1, \kappa\}$, where

$$
\begin{aligned}
& \kappa=\frac{\theta}{2}+\frac{\sqrt{\mu}}{2 \gamma} \in O_{L}, \\
& \theta=0,1, \sqrt{c}, 1+\sqrt{c}, \frac{1+\sqrt{c}}{2} \text { or } \frac{-1+\sqrt{c}}{2}
\end{aligned}
$$

depending on congruence conditions involving $a, b, c$,

$\mu=a+b \sqrt{c}$,

* Research supported by Natural Sciences and Engineering Research Council of Canada Grant A-7233.

1991 Mathematics Subject Classification: 11R16, 11R04.

Key words and phrases. quartic fields with quadratic subfields, normal relative integral basis.

Received August 3, 1994 ; revised March 22, 1996. 
$\mu O_{K}=R S^{2}$, where $R$ and $S$ are

integral ideals of $O_{K}$ with $R$ squarefree, $d(L / K)=R T^{2}$, where $T^{2}=O_{K}, 2 O_{K}$,

$4 O_{K},\left\langle 2, \frac{1}{2}(1+\sqrt{c})\right\rangle^{2}$ or $\left\langle 2, \frac{1}{2}(1-\sqrt{c})\right\rangle^{2}$

depending on congruence conditions involving $a, b, c$, $S=T\langle\gamma\rangle$, where $\gamma \in K \backslash\{0\}$.

It is convenient to define the nonnegative integer $r$ by

$$
2^{r} \| a^{2}-b^{2} c
$$

and the integers $a^{\prime}$ and $b^{\prime}$ by

$$
\mu / \gamma^{2}= \begin{cases}\left(a^{\prime}+b^{\prime} \sqrt{c}\right) / 2, & \text { if } c \equiv 1(\bmod 4), \\ a^{\prime}+b^{\prime} \sqrt{c}, & \text { if } c \equiv 2,3(\bmod 4) .\end{cases}
$$

When $c \equiv 1(\bmod 4)$, as $\mu / \gamma^{2} \in O_{K}, a^{\prime}, b^{\prime}$ are integers with $a^{\prime} \equiv b^{\prime}(\bmod 2)$.

If $c>0$, we let $\varepsilon_{c}$ denote the fundamental unit $(>1)$ of $K=Q(\sqrt{c})$, and set

$$
N(c)=\text { norm of } \varepsilon_{c}= \pm 1
$$

and

$$
F(c)= \begin{cases}+1, & \text { if } \varepsilon_{c}=(t+u \sqrt{c}) / 2 \text { for odd integers } t \text { and } u, \\ -1, & \text { if } \varepsilon_{c}=t+u \sqrt{c} \quad \text { for integers } t \text { and } u\end{cases}
$$

In Section 2 we prove the following theorem, which extends a theorem of Kawamoto [5, Theorem 7].

THEOREM 1. Let $a, b, c$ be integers with $(a, b)$ squarefree, $c$ squarefree, and $a+b \sqrt{c}$ not a square in $Q(\sqrt{c})$. Set $L=Q(\sqrt{c}, \sqrt{a+b \sqrt{c}})$ and $K=Q(\sqrt{c})$. Suppose $L$ has a relative integral basis over $K$. Define $\mu, \gamma, r, a^{\prime}, b^{\prime}, N(c), F(c)$, $t$ and $u$ as in (1.3)-(1.10). Then $L$ possesses a NRIB over $K$ only in the cases listed below. In each case an integer $\omega$ of $K$ is given so that $\left\{\omega, \omega^{\prime}\right\}$ is a NRIB. [For compactness we write $x \equiv y(m)$ for $x \equiv y(\bmod m)$.]

(i ) $\quad a \equiv 1(2), \quad b \equiv 0(2), \quad a+b \equiv 1(4), \quad a^{\prime} \equiv 1(4)$,

(ii) $\quad a \equiv 1(2), \quad b \equiv 0(2), \quad a+b \equiv 1(4), \quad a^{\prime} \equiv 3(4), \quad c>0, \quad N(c)=-1$,

(iii) $a \equiv 2(4), \quad b \equiv 0(4), \quad a+b \equiv c(8), \quad a^{\prime} \equiv 1(4)$,

(iv) $\quad a \equiv 2(4), \quad b \equiv 0(4), \quad a+b \equiv c(8), \quad a^{\prime} \equiv 3(4), \quad c>0, \quad N(c)=-1$.

$$
\omega=\frac{1}{2}+\frac{\sqrt{\mu}}{2 \gamma} \text { (i) (iii) } \omega=\frac{t+u \sqrt{c}}{2}+\frac{\sqrt{\mu}}{2 \gamma} \text { (ii) (iv) }
$$


$c \equiv 3(4)$

(i ) $\quad a \equiv 1(2), \quad b \equiv 0(4), \quad a^{\prime} \equiv 1(4)$,

(ii) $\quad a \equiv 1(2), \quad b \equiv 0(4), \quad a^{\prime} \equiv 3(4), \quad c=-1$,

(iii) $\quad a \equiv 1(2), \quad b \equiv 0(4), \quad a^{\prime} \equiv 3(4), \quad c>0, t \equiv 0(2), u \equiv 1(2)$,

(iv) $a \equiv 0(4), \quad b \equiv 2(4), \quad a \equiv c+1(8), \quad a^{\prime} \equiv 1(4)$,

(v) $a \equiv 0(4), \quad b \equiv 2(4), \quad a \equiv c+1(8), \quad a^{\prime} \equiv 3(4), \quad c=-1$,

(vi) $\quad a \equiv 0(4), \quad b \equiv 2(4), \quad a \equiv c+1(8), \quad a^{\prime} \equiv 3(4), c>0, t \equiv 0(2), u \equiv 1(2)$.

$$
\begin{aligned}
& \omega=\frac{1}{2}+\frac{\sqrt{\mu}}{2 \gamma} \text { (i) (iv) } \omega=\frac{\sqrt{c}}{2}+\frac{\sqrt{\mu}}{2 \gamma} \text { (ii) (v) } \\
& \omega=\frac{t+u \sqrt{c}}{2}+\frac{\sqrt{\mu}}{2 \gamma} \text { (iii) (vi) }
\end{aligned}
$$

$c \equiv 5(8)$

(i ) $a \equiv 1(2), \quad b \equiv 0(2), \quad a+b \equiv 1(4), \quad a^{\prime} \equiv b^{\prime} \equiv 0(2)$,

(ii) $a \equiv 1(2), \quad b \equiv 0(2), \quad a+b \equiv 1(4), \quad a^{\prime} \equiv b^{\prime} \equiv 1(2), c=-3$,

(iii) $a \equiv 1(2), \quad b \equiv 0(2), \quad a+b \equiv 1(4), \quad a^{\prime} \equiv b^{\prime} \equiv 1(2), c>0, F(c)=1$,

(iv) $a \equiv 6(8), \quad b \equiv 2(4), \quad a-b-c \equiv 3$ or $15(16), \quad c=-3$,

(v) $a \equiv 6(8), \quad b \equiv 2(4), \quad a-b-c \equiv 3$ or $15(16), \quad c>0, F(c)=1$.

$$
\begin{aligned}
& \omega=\frac{1}{2}+\frac{\sqrt{\mu}}{2 \gamma} \text { (i) } \omega=\frac{1+(-1)^{\left(1-b^{\prime}\right) / 2} \sqrt{c}}{4}+\frac{\sqrt{\mu}}{2 \gamma} \text { (ii) (iv) } \\
& \omega=\frac{t+(-1)^{\left(t-b^{\prime} u\right) / 2} u \sqrt{c}}{4}+\frac{\sqrt{\mu}}{2 \gamma} \text { (iii) (v) }
\end{aligned}
$$

$c \equiv 1(8)$

(i ) $a \equiv 1(2), \quad b \equiv 0(2), \quad a+b \equiv 1(4)$,

(ii) $a \equiv 2(8), \quad b \equiv 2(4), \quad r$ (even) $\geqq 6, \quad\left(a^{2}-b^{2} c\right) / 2^{r} \equiv 1(4)$.

$$
\omega=\frac{1}{2}+\frac{\sqrt{\mu}}{2 \gamma} \text { (i) (ii) }
$$

In Sections 3, 4 and 5 we investigate the special cases when $L$ is cyclic, bicyclic, and pure respectively. We determine when the existence of a RIB and a squarefree relative discriminant are both necessary and sufficient for the existence of a NRIB.

THEOREM 2. If $L$ is a cyclic quartic field with quadratic subfield $K$, then $L / K$ has a NRIB if and only if $L / K$ has a $R I B$ and $d(L / K)$ is squarefree.

THEOREM 3. Let $c$ be a squarefree integer, and set $K=Q(\sqrt{c})$. Let $L$ be a bicyclic quartic field containing $K$. Then $L=Q(\sqrt{c}, \sqrt{a})$ for some squarefree integer $a$ with $a \neq c$. As $L=Q\left(\sqrt{c}, \sqrt{a c /(a, c)^{2}}\right)$, we can choose between $a$ and $a c /(a, c)^{2}$ when $c \neq-1$ so that $c+a$. 
If $c=-3,-1$, or $c>0, N(c)=-1$, then

$L / K$ has a NRIB $\Longleftrightarrow L / K$ has a RIB and $d(L / K)$ is squarefree.

If $c<-3$ then

$L / K$ has a NRIB $\Longleftrightarrow L / K$ has a $R I B, d(L / K)$ is squarefree, and $a \equiv 1(\bmod 4)$.

If $c>0$ and $N(c)=1$ then

$L / K$ has a NRIB $\Longleftrightarrow L / K$ has a $R I B, d(L / K)$ is squarefree, and

$$
\left\{\begin{array}{c}
(a, c)=1, a \equiv 1(\bmod 4) \\
\text { or } \\
(a, c)=1, c \equiv 3(\bmod 4), a \equiv 3(\bmod 4), t \equiv 0(\bmod 2), u \equiv 1(\bmod 2) \\
\text { or } \\
(a, c) \neq 1, c \equiv 1(\bmod 4) \\
\text { or } \\
(a, c) \neq 1, c \neq 1(\bmod 4), \frac{a t}{(a, c)} \equiv 1(\bmod 4) .
\end{array}\right.
$$

THEOREM 4. If $L$ is a pure quartic field then $L=Q(\sqrt{b \sqrt{c}})$, where $b$ and $c$ are squarefree integers with $(b, c) \neq( \pm 2,-1)$ and $c+b$ if $c \neq-1$. Set $K=Q(\sqrt{c})$. Then

$L / K$ has a NRIB $\Longleftrightarrow L / K$ has a $R I B$ and $d(L / K)$ is squarefree.

Kawamoto [5, Propositions 10 and 11] has different formulations of Theorems 2 and 3. Massy [6], [7] has given a necessary and sufficient condition for a quadratic field $K$ to be a subfield of a cyclic quartic field $L$ possessing a NRIB over $K$.

\section{Proof of Theorem 1}

Let $L=Q(\sqrt{c}, \sqrt{a+b \sqrt{c}})$ and $K=Q(\sqrt{c})$, where $a, b, c$ are integers such that $(a, b)$ and $c$ are squarefree, and $a+b \sqrt{c} \notin K^{2}$. We suppose that $L$ possesses a RIB over $K$, and take the RIB in the form $\{1, \kappa\}$, where $\kappa$ is given by $(1.1)$.

Before proving Theorem 1, we prove four lemmas. We denote the group of units of $O_{K}$ by $U_{K}$.

LEMMA 1. Let the fields $L$ and $K$ be as specified above. If the relative discriminant $d(L / K)$ is not squarefree, then $L / K$ does not possess a NRIB.

Proof. Let $\{1, \kappa\}$ be the RIB for $L / K$ specified above, and suppose that $L / K$ possesses a NRIB, say, $\left\{\alpha+\beta \kappa, \alpha+\beta \kappa^{\prime}\right\}$, where $\alpha, \beta \in O_{K}$ and $\kappa^{\prime}$ denotes 
the conjugate of $\kappa$ over $K$. As $\left\{\alpha+\beta \kappa, \alpha+\beta \kappa^{\prime}\right\}$ is a RIB for $L / K$, there exist $\lambda, \phi \in O_{K}$ with

$$
1=\lambda(\alpha+\beta \kappa)+\phi\left(\alpha+\beta \kappa^{\prime}\right) .
$$

Taking the conjugates of (2.1) over $K$, we obtain

$$
1=\lambda\left(\alpha+\beta \kappa^{\prime}\right)+\phi(\alpha+\beta \kappa) .
$$

From (2.1) and (2.2), we see that $\lambda=\phi$. Then (2.1) gives $1=\lambda\left(2 \alpha+\beta\left(\kappa+\kappa^{\prime}\right)\right)$, so that $2 \alpha+\beta\left(\kappa+\kappa^{\prime}\right) \in U_{K}$. Next, we have

$$
\begin{aligned}
d(L / K) & =\left|\begin{array}{ll}
\alpha+\beta \kappa & \alpha+\beta \kappa^{\prime} \\
\alpha+\beta \kappa^{\prime} & \alpha+\beta \kappa
\end{array}\right|^{2} O_{K} \\
& =\left((\alpha+\beta \kappa)^{2}-\left(\alpha+\beta \kappa^{\prime}\right)^{2}\right)^{2} O_{K} \\
& =\beta^{2}\left(\kappa-\kappa^{\prime}\right)^{2}\left(2 \alpha+\beta\left(\kappa+\kappa^{\prime}\right)\right)^{2} O_{K} \\
& =\beta^{2}\left(\kappa-\kappa^{\prime}\right)^{2} O_{K} .
\end{aligned}
$$

Now suppose that $d(L / K)$ is not squarefree. Thus there exists a prime ideal $P$ of $O_{K}$ with $P^{2} \mid d(L / K)$, so that

$$
P^{2} \mid \beta^{2}\left(\kappa-\kappa^{\prime}\right)^{2} O_{K} .
$$

Let $\mathscr{P}$ be a prime ideal in $O_{L}$ lying above $P$. Then, from (2.3), we see that

$$
\mathscr{D} \mid \beta\left(\kappa-\kappa^{\prime}\right) O_{L}
$$

From (1.4) and (1.5), we deduce that $P \mid 2 O_{K}$, so that $\mathscr{Q} \mid 2 O_{L}$. Hence we have

$$
\mathscr{P} \mid\left(\beta\left(\kappa-\kappa^{\prime}\right)+2\left(\alpha+\beta \kappa^{\prime}\right)\right) O_{L},
$$

contradicting that $2 \alpha+\beta\left(\kappa+\kappa^{\prime}\right) \in U_{K}$.

LEMMA 2. Let the fields $L$ and $K$ be as specified above with relative integral basis $\{1, \kappa\}$, where $\kappa$ is defined in (1.1). Then $L / K$ has a NRIB if and only if there exists $\lambda \in U_{K}$ such that

$$
2 \mid \lambda-\theta
$$

where $\theta$ is given by (1.2). When (2.4) holds, a NRIB for $L / K$ is

$$
\left\{\frac{\lambda}{2}+\frac{\sqrt{\mu}}{2 \gamma}, \frac{\lambda}{2}-\frac{\sqrt{\mu}}{2 \gamma}\right\} \text {. }
$$

Proof. Suppose $L / K$ has a NRIB, say, $\left\{\alpha+\beta \kappa, \alpha+\beta \kappa^{\prime}\right\}$. Then, exactly as in the proof of Lemma 1 , we deduce that $\varepsilon=2 \alpha+\beta\left(\kappa+\kappa^{\prime}\right)=2 \alpha+\beta \theta \in U_{K}$. As $\left\{\alpha \varepsilon^{-1}+\beta \varepsilon^{-1} \kappa, \alpha \varepsilon^{-1}+\beta \varepsilon^{-1} \kappa^{\prime}\right\}$ is also a NRIB for $L / K$, we may take $\varepsilon=1$ without loss of generality, so that 


$$
2 \alpha+\beta \theta=1 .
$$

As $\left\{\alpha+\beta \kappa, \alpha+\beta \kappa^{\prime}\right\}$ is a RIB for $L / K$, there exist $\rho, \tau \in O_{K}$ such that

$$
\kappa=\rho(\alpha+\beta \kappa)+\tau\left(\alpha+\beta \kappa^{\prime}\right),
$$

and so, by (1.1), we have

$$
\frac{\theta}{2}+\frac{\sqrt{\mu}}{2 \gamma}=\rho\left(\alpha+\beta \frac{\theta}{2}+\beta \frac{\sqrt{\mu}}{2 \gamma}\right)+\tau\left(\alpha+\beta \frac{\theta}{2}-\beta \frac{\sqrt{\mu}}{2 \gamma}\right) .
$$

Equating coefficients of $\sqrt{\mu} / 2 \gamma$ in (2.6), we obtain $1=(\rho-\tau) \beta$, showing that $\beta \in U_{K}$. We define $\lambda \in U_{K}$ by $\lambda=1 / \beta$, and, from (2.5), we deduce that $2 \mid \lambda-\theta$, and a NRIB for $L / K$ is

$$
\begin{aligned}
\left\{\lambda(\alpha+\beta \kappa), \lambda\left(\alpha+\beta \kappa^{\prime}\right)\right\} & =\left\{\lambda \alpha+\kappa, \lambda \alpha+\kappa^{\prime}\right\} \\
& =\left\{\frac{\lambda-\theta}{2}+\frac{\theta}{2}+\frac{\sqrt{\mu}}{2 \gamma}, \frac{\lambda-\theta}{2}+\frac{\theta}{2}-\frac{\sqrt{\mu}}{2 \gamma}\right\} \\
& =\left\{\frac{\lambda}{2}+\frac{\sqrt{\mu}}{2 \gamma}, \frac{\lambda}{2}-\frac{\sqrt{\mu}}{2 \gamma}\right\} .
\end{aligned}
$$

Conversely suppose that $\lambda \in U_{K}$ with $2 \mid \lambda-\theta$. Then we have $\alpha=(\lambda-\theta) / 2 \in O_{K}$. We claim that $\{\lambda / 2+\sqrt{\mu} / 2 \gamma, \lambda / 2-\sqrt{\mu} / 2 \gamma\}=\left\{\alpha+\kappa, \alpha+\kappa^{\prime}\right\}$ is a NRIB. This is clear as

and

$$
1=\frac{1}{\lambda}(\alpha+\kappa)+\frac{1}{\lambda}\left(\alpha+\kappa^{\prime}\right)
$$

$$
\kappa=\left(\frac{\lambda+\theta}{2 \lambda}\right)(\alpha+\kappa)-\left(\frac{\lambda-\theta}{2 \lambda}\right)\left(\alpha+\kappa^{\prime}\right) .
$$

The next lemma summarizes some elementary properties of the form of the units of $O_{K}$ when $c>0$. The proof of the lemma is an easy exercise in elementary number theory.

LEMMA 3. Let $c$ be a positive squarefree integer.

If $c \equiv 2(\bmod 4)$ then $F(c)=-1, N(c)= \pm 1$, and every unit of $O_{K}$ is of the form $x+y \sqrt{c}$, where the integers $x$ and $y$ satisfy

$$
\begin{aligned}
& x \equiv 1(\bmod 2), \quad y \equiv 0(\bmod 2), \quad \text { if } x^{2}-c y^{2}=1, \\
& x \equiv 1(\bmod 2), \quad y \equiv 1(\bmod 2), \quad \text { if } x^{2}-c y^{2}=-1 .
\end{aligned}
$$

If $c \equiv 3(\bmod 4)$ then $F(c)=-1, N(c)=1$, and every unit of $O_{K}$ is of the form $x+y \sqrt{c}$, where the integers $x$ and $y$ satisfy 
or

$$
x \equiv 0(\bmod 2), \quad y \equiv 1(\bmod 2)
$$

$$
x \equiv 1(\bmod 2), \quad y \equiv 0(\bmod 2) \text {. }
$$

If $c \equiv 5(\bmod 8)$ and $F(c)=1$, then $N(c)= \pm 1$ and every unit of $O_{K}$ is of the form $(x+y \sqrt{c}) / 2$, where the integers $x$ and $y$ satisfy

or

$$
x \equiv y \equiv 1(\bmod 2)
$$

or

$$
x \equiv 0(\bmod 4), \quad y \equiv 2(\bmod 4), \quad x^{2}-c y^{2}=-4,
$$$$
x \equiv 2(\bmod 4), \quad y \equiv 0(\bmod 4), \quad x^{2}-c y^{2}=4 \text {. }
$$

If $c \equiv 5(\bmod 8)$ and $F(c)=-1$, then $N(c)= \pm 1$ and every unit of $O_{K}$ is of the form $x+y \sqrt{c}$, where the integers $x$ and $y$ satisfy

or

$$
x \equiv 0(\bmod 2), \quad y \equiv 1(\bmod 2), \quad \text { if } x^{2}-c y^{2}=-1,
$$

$$
x \equiv 1(\bmod 2), \quad y \equiv 0(\bmod 2), \quad \text { if } x^{2}-c y^{2}=1 \text {. }
$$

If $c \equiv 1(\bmod 8)$ then $F(c)=-1, N(c)= \pm 1$, and every unit of $O_{K}$ is of the form $x+y \sqrt{c}$, where the integers $x$ and $y$ satisfy

$$
\begin{aligned}
& x \equiv 1(\bmod 2), \quad y \equiv 0(\bmod 4), \quad \text { if } x^{2}-c y^{2}=1, \\
& x \equiv 0(\bmod 4), \quad y \equiv 1(\bmod 2), \quad \text { if } x^{2}-c y^{2}=-1 .
\end{aligned}
$$

In Lemma 4 we make use of Lemma 3 to determine $\lambda \in U_{K}$ satisfying (2.4) when such $\lambda$ exists.

LEMMA 4. Let $c$ be a squarefree integer.

If $c \equiv 2(\bmod 4)$ then $\theta=0,1, \sqrt{c}$ or $1+\sqrt{c}$, and there exists $\lambda \in U_{K}$ with $2 \mid \lambda-\theta$ if and only if

or

$$
\theta=1 \quad(\lambda=1)
$$

$$
\theta=1+\sqrt{c}, \quad c>0, \quad N(c)=-1 \quad\left(\lambda=\varepsilon_{c}\right) .
$$

If $c \equiv 3(\bmod 4)$ then $\theta=0,1, \sqrt{c}$ or $1+\sqrt{c}$, and there exists $\lambda \in U_{K}$ with $2 \mid \lambda-\theta$ if and only if

or

$$
\theta=1 \quad(\lambda=1)
$$

$$
\theta=\sqrt{c}, \quad c>0, \quad t \equiv 0(\bmod 2), \quad u \equiv 1(\bmod 2) \quad\left(\lambda=\varepsilon_{c}\right)
$$


or

$$
\theta=\sqrt{c}, \quad c=-1 \quad(\lambda=\sqrt{-1})
$$

If $c \equiv 5(\bmod 8)$ then $\theta=0,1$, or $\left(b^{\prime}+\sqrt{c}\right) / 2$, and there exists $\lambda \in U_{K}$ with $2 \mid \lambda-\theta$ if and only if

or

$$
\theta=1 \quad(\lambda=1)
$$

$$
\theta=\frac{b^{\prime}+\sqrt{c}}{2}, \quad c=-3 \quad\left(\lambda=\frac{1+(-1)^{\left(1-b^{\prime}\right) / 2} \sqrt{-3}}{2}\right)
$$

or

$$
\theta=\frac{b^{\prime}+\sqrt{c}}{2}, \quad c>0, \quad \text { and } \quad F(c)=1 \quad\left(\lambda=\frac{t+(-1)^{\left(t-b^{\prime} u\right) / 2} u \sqrt{c}}{2}\right) .
$$

If $c \equiv 1(\bmod 8)$ then $\theta=0,1,(1+\sqrt{c}) / 2$, or $(-1+\sqrt{c}) / 2$, and there exists $\lambda \in U_{K}$ with $2 \mid \lambda-\theta$ if and only if

$$
\theta=1 \quad(\lambda=1)
$$

Proof. The values of $\theta$ corresponding to the residue class of $c$ modulo 4 or 8 follow from [9, Theorem 2]. The remaining assertions of the lemma follow easily from Lemma 3.

We are now ready to prove Theorem 1.

Proof of Theorem 1. Recall that we are assuming that $L / K$ has the RIB $\{1, \kappa\}$. Suppose further that $L / K$ has a NRIB. By Lemma $1 d(L / K)$ is squarefree. Appealing to [9, Theorem 1] $a, b, c$ must fall into one of the following cases:

Case 1: $a \equiv 1(\bmod 2), b \equiv 0(\bmod 2), c \equiv 2(\bmod 4), a+b \equiv 1(\bmod 4)$, Case 2: $a \equiv 2(\bmod 4), b \equiv 0(\bmod 4), c \equiv 2(\bmod 4), a+b \equiv c(\bmod 8)$,

Case 3: $a \equiv 1(\bmod 2), b \equiv 0(\bmod 4), c \equiv 3(\bmod 4)$,

Case 4: $a \equiv 0(\bmod 4), b \equiv 2(\bmod 4), c \equiv 3(\bmod 4), a \equiv c+1(\bmod 8)$,

Case 5: $a \equiv 1(\bmod 2), b \equiv 0(\bmod 2), c \equiv 5(\bmod 8), a+b \equiv 1(\bmod 4)$,

Case $6: a \equiv 6(\bmod 8), b \equiv 2(\bmod 4), c \equiv 5(\bmod 8), a-b-c \equiv 3$ or $15(\bmod 16)$,

Case $7: a \equiv 1(\bmod 2), b \equiv 0(\bmod 2), c \equiv 1(\bmod 8), a+b \equiv 1(\bmod 4)$,

Case $8: a \equiv 2(\bmod 8), b \equiv 2(\bmod 8), c \equiv 1(\bmod 8), r($ even $) \geqq 6$,

Case 9: $a \equiv 2(\bmod 8), b \equiv 6(\bmod 8), c \equiv 1(\bmod 8), r($ even $) \geqq 6$,

$$
\left(a^{2}-b^{2} c\right) / 2^{r} \equiv 1(\bmod 4),
$$

$$
\left(a^{2}-b^{2} c\right) / 2^{r} \equiv 1(\bmod 4) \text {. }
$$

We emphasize that if $a, b, c$ do not satisfy one of Cases 1 to 9 then $d(L / K)$ is not squarefree and $L / K$ does not possess a NRIB. We now examine each of the above cases making use of Lemma 4 to determine the additional constraints on $a, b, c$ in order for $L / K$ to have a NRIB. 
Cases 1 and 2. By [9, Theorem 2] we have

$$
\theta= \begin{cases}1, & \text { if } a^{\prime} \equiv 1(\bmod 4), \\ 1+\sqrt{c}, & \text { if } a^{\prime} \equiv 3(\bmod 4) .\end{cases}
$$

Thus, by Lemmas 2 and $4, L / K$ has NRIB in this case if and only if

$$
a^{\prime} \equiv 1(\bmod 4)
$$

or

$$
a^{\prime} \equiv 3(\bmod 4), \quad c>0, \quad N(c)=-1 .
$$

The NRIB's are respectively

and

$$
\left\{\frac{1}{2}+\frac{\sqrt{\mu}}{2 \gamma}, \frac{1}{2}-\frac{\sqrt{\mu}}{2 \gamma}\right\}
$$

$$
\left\{\frac{t+u \sqrt{c}}{2}+\frac{\sqrt{\mu}}{2 \gamma}, \frac{t+u \sqrt{c}}{2}-\frac{\sqrt{\mu}}{2 \gamma}\right\}
$$

Cases 3 and 4. By [9, Theorem 2] we have

$$
\theta= \begin{cases}1, & \text { if } a^{\prime} \equiv 1(\bmod 4), \\ \sqrt{c}, & \text { if } a^{\prime} \equiv 3(\bmod 4) .\end{cases}
$$

Then, by Lemmas 2 and $4, L / K$ has a NRIB in this case if and only if

or

$$
a^{\prime} \equiv 1(\bmod 4)
$$

or

$$
a^{\prime} \equiv 3(\bmod 4), \quad c=-1,
$$

$$
a^{\prime} \equiv 3(\bmod 4), \quad c>0, \quad t \equiv 0(\bmod 2), \quad u \equiv 1(\bmod 2) .
$$

The NRIB's are respectively

and

$$
\begin{aligned}
& \left\{\frac{1}{2}+\frac{\sqrt{\mu}}{2 \gamma}, \frac{1}{2}-\frac{\sqrt{\mu}}{2 \gamma}\right\}, \\
& \left\{\frac{\sqrt{c}}{2}+\frac{\sqrt{\mu}}{2 \gamma}, \frac{\sqrt{c}}{2}-\frac{\sqrt{\mu}}{2 \gamma}\right\},
\end{aligned}
$$

$$
\left\{\frac{t+u \sqrt{c}}{2}+\frac{\sqrt{\mu}}{2 \gamma}, \frac{t+u \sqrt{c}}{2}-\frac{\sqrt{\mu}}{2 \gamma}\right\} .
$$

Case 5. By [9, Theorem 2] we have

$$
\theta= \begin{cases}1, & \text { if } a^{\prime} \equiv b^{\prime} \equiv 0(\bmod 2), \\ \frac{b^{\prime}+\sqrt{c}}{2}, & \text { if } a^{\prime} \equiv b^{\prime} \equiv 1(\bmod 2) .\end{cases}
$$


Then, by Lemmas 2 and $4, L / K$ has a NRIB in this case if and only if

or

$$
a^{\prime} \equiv b^{\prime} \equiv 0(\bmod 2)
$$

or

$$
a^{\prime} \equiv b^{\prime} \equiv 1(\bmod 2), \quad c=-3
$$

$$
a^{\prime} \equiv b^{\prime} \equiv 1(\bmod 2), \quad c>0, \quad F(c)=1 .
$$

The NRIB's are respectively

$$
\begin{aligned}
& \left\{\frac{1}{2}+\frac{\sqrt{\mu}}{2 \gamma}, \frac{1}{2}-\frac{\sqrt{\mu}}{2 \gamma}\right\}, \\
& \left\{\frac{1+(-1)^{\left(1-b^{\prime}\right) / 2} \sqrt{c}}{4}+\frac{\sqrt{\mu}}{2 \gamma}, \frac{1+(-1)^{\left(1-b^{\prime}\right) / 2} \sqrt{c}}{4}-\frac{\sqrt{\mu}}{2 \gamma}\right\}, \\
& \left\{\frac{t+(-1)^{\left(t-b^{\prime} u\right) / 2} u \sqrt{c}}{4}+\frac{\sqrt{\mu}}{2 \gamma}, \frac{t+(-1)^{\left(t-b^{\prime} u\right) / 2} u \sqrt{c}}{4}-\frac{\sqrt{\mu}}{2 \gamma}\right\} .
\end{aligned}
$$

Case 6. By [9, Theorem 2] we have

$$
\theta=\frac{b^{\prime}+\sqrt{c}}{2} .
$$

Thus, by Lemmas 2 and $4, L / K$ has a NRIB in this case if and only if

or

$$
a^{\prime} \equiv b^{\prime} \equiv 1(\bmod 2), \quad c=-3
$$

$$
a^{\prime} \equiv b^{\prime} \equiv 1(\bmod 2), \quad c>0, \quad F(c)=1 .
$$

The NRIB's are respectively

$$
\begin{aligned}
& \left\{\frac{1+(-1)^{\left(1-b^{\prime}\right) / 2} \sqrt{c}}{4}+\frac{\sqrt{\mu}}{2 \gamma}, \frac{1+(-1)^{\left(1-b^{\prime}\right) / 2} \sqrt{c}}{4}-\frac{\sqrt{\mu}}{2 \gamma}\right\}, \\
& \left\{\frac{t+(-1)^{\left(t-b^{\prime} u\right) / 2} u \sqrt{c}}{4}+\frac{\sqrt{\mu}}{2 \gamma}, \frac{t+(-1)^{\left(t-b^{\prime} u\right) / 2} u \sqrt{c}}{4}-\frac{\sqrt{\mu}}{2 \gamma}\right\} .
\end{aligned}
$$

Cases 7, 8, 9. By [9, Theorem 2] we have $\theta=1$. Thus, by Lemmas 2 and $4, L / K$ has a NRIB namely,

$$
\left\{\frac{1}{2}+\frac{\sqrt{\mu}}{2 \gamma}, \frac{1}{2}-\frac{\sqrt{\mu}}{2 \gamma}\right\} .
$$

\section{3. $L$ cyclic: Proof of Theorem 2}

Let $L$ be a cyclic quartic field with unique quadratic subfield $K$, and assume that $L / K$ has a RIB. By Lemma 1 we know that if $d(L / K)$ is not squarefree then $L / K$ does not possess a NRIB. Thus to complete the proof it suffices to prove that if $d(L / K)$ is squarefree then $L / K$ has a NRIB. It is known (see 
[8]) that $L$ may be taken in the form $L=Q(\sqrt{A(D+B \sqrt{D})})$, where $A$ is squarefree and odd, $D=B^{2}+C^{2}$ is squarefree $(B>0, C>0)$, and $(A, D)=1$. Then, appealing to $[8$, Lemma 2$]$, we see that $d(L / K)$ squarefree implies

$$
D \equiv 1(\bmod 4), \quad B \equiv 0(\bmod 2), \quad A+B \equiv 1(\bmod 4) \text {. }
$$

Further, by [8, Theorem 3$]$, as $L / K$ has a RIB, we can take the RIB as

$$
\left\{1, \frac{1}{2}(1+\sqrt{A(D+B \sqrt{D})})\right\} \text {. }
$$

Thus $L$ possesses a NRIB over $K$, namely,

$$
\left\{\frac{1}{2}(1-\sqrt{A(D+B \sqrt{D})}), \frac{1}{2}(1+\sqrt{A(D+B \sqrt{D})})\right\} .
$$

\section{4. $L$ bicyclic: Proof of Theorem 3}

If $L / K$ has a NRIB then clearly $L / K$ has a RIB and, by Lemma $1, d(L / K)$ is squarefree.

Now suppose that $L / K$ has a RIB and $d(L / K)$ is squarefree. There are nine possibilities for the pair $(c, a)(\bmod 4)$. The second assumption by $[9$, Theorem 1] eliminates four of these and leaves only the five possibilities

$$
(c, a) \equiv(1,1),(2,1),(2,2)(\text { with } a \equiv c(\bmod 8)),(3,1),(3,3)(\bmod 4) .
$$

Further, the first assumption by [9, Theorem 2] guarantees the existence of an element $\gamma$ in $O_{K}$ with $S=\gamma O_{K}$. Recalling that the only primes which ramify in $K$ are the odd prime divisors of $c$ and the prime 2 if $c \neq \equiv 1(\bmod 4)$, we see from (1.4) that $S^{2}=(a, c) O_{K}$. Thus

$$
\gamma^{2}=(a, c) \theta \text {, for some unit } \theta \text { of } O_{K} .
$$

It is now convenient to treat cases.

$c=-3$. From $(4.1)$ we have $a \equiv 1(\bmod 4)$, and by Theorem $1(c \equiv 5(\bmod 8)$, (i), (ii)) $L / K$ has a NRIB.

$c=-1$. Here $\theta= \pm 1$ or $\pm i$. From $(4.1)$ we have $a \equiv 1(\bmod 2)$. Further $(a, c)=1$ as $\gamma^{2}=(a, c) \theta$ cannot hold with $\theta= \pm i$. Thus $\theta= \pm 1, \gamma^{2}= \pm 1, a^{\prime}+b^{\prime} i$ $=a / \gamma^{2}= \pm a$, so $a^{\prime} \equiv 1(\bmod 2)$. Hence by Theorem $1(c \equiv 3(\bmod 4)$, (i), (ii)) $L / K$ has a NRIB.

$c>0, N(c)=-1$. As $N(c)=-1$, we have $c \neq \equiv 3(\bmod 4)$. Thus, by (4.1), we have $(c, a) \equiv(1,1),(2,1)$ or $(2.2)(\bmod 4)$. Clearly, from $(4.2)$, we see that we may assume without loss of generality that $\theta= \pm 1$ or $\theta= \pm \varepsilon_{c}$.

When $c \equiv 2(\bmod 4), \theta$ is of the form $x+y \sqrt{c}$ with $x$ odd, so from $a^{\prime}+b^{\prime} \sqrt{c}=a /((a, c) \theta)$, we see that $a^{\prime}$ is odd. Hence, by Theorem $1(c \equiv 2$ $(\bmod 4),(\mathrm{i})-(\mathrm{iv})), L / K$ has a NRIB.

When $c \equiv 1(\bmod 8)$, we have $a \equiv 1(\bmod 4)$, and by Theorem $1(c \equiv 1(\bmod 8)$, (i)) $L / K$ has a NRIB. 
When $c \equiv 5(\bmod 8)$ we must examine $\theta$ more closely. Clearly $\theta=\gamma^{2} /(a, c)$ $>0$ so that $\theta=1$ or $\varepsilon_{c}$. Further

$$
N(\theta)=N(\gamma)^{2} /(a, c)^{2}>0
$$

so that $\theta \neq \varepsilon_{c}$ as $N\left(\varepsilon_{c}\right)=-1$. Hence $\theta=1$, and $\gamma^{2}=(a, c)$. As $\gamma \in O_{K}$ we have $\gamma=(r+s \sqrt{c}) / 2$, where $r, s$ are integers with $r \equiv s(\bmod 2)$. Thus

$$
r^{2}+s^{2} c=4(a, c), \quad 2 r s=0 .
$$

If $r=0$ then $s^{2} c=4(a, c)$ so $c \mid a$, a contradiction. If $s=0$ then $r^{2}=4(a, c)$ so $(r / 2)^{2}=(a, c)$. But $(a, c)$ is squarefree, so $r / 2= \pm 1,(a, c)=1$, and $r^{2}=1$. Thus $\left(a^{\prime}+b^{\prime} \sqrt{c}\right) / 2=a$, so $a^{\prime} \equiv b^{\prime} \equiv 0(\bmod 2)$, and by Theorem $1(c \equiv 5(\bmod 8)$, (i $\left.)\right)$ $L / K$ has a NRIB.

$c<-3$. Here $\theta= \pm 1$. From (4.2) we have $\gamma^{2}= \pm(a, c)$. We show that the plus sign must hold and $(a, c)=1$, for otherwise (remembering that $c$ and $(a, c)$ are squarefree) we have $[Q(\sqrt{ \pm(a, c)}): Q]=2$ and $\sqrt{ \pm(a, c)}=\gamma \in Q(\sqrt{c})$, so $c=-(a, c)$ and thus $c \mid a$, a contradiction. Hence $\gamma^{2}=(a, c)=1$. Note that this rules out the case $c \equiv a \equiv 2(\bmod 4)$. (There is no RIB in this case.) Now by (1.8) we have

$$
a^{\prime}+b^{\prime} \sqrt{c}= \begin{cases}a, & \text { if } c \neq 1(\bmod 4), \\ 2 a, & \text { if } c \equiv 1(\bmod 4) .\end{cases}
$$

From Theorem 1 (examining cases), we see that $L / K$ possesses a NRIB only when $a \equiv 1(\bmod 4)$.

$c>0, N(c)=1$. From (4.2) we see without loss of generality that $\theta= \pm 1$ or $\theta= \pm \varepsilon_{c}$. As $\theta=\gamma^{2} /(a, c)>0$, we have $\theta=1$ or $\theta=\varepsilon_{c}$. If $(a, c) \neq 1$ we show that $\theta=\varepsilon_{c} \quad$ Otherwise $\theta=1,[Q(\sqrt{ }(\overline{a, c})): Q]=2$ and $\sqrt{(a, c)}=\gamma \in Q(\sqrt{c})$, so $(a, c)=c$ contradicting $c+a$. If $(a, c)=1$ we show that $\theta=1$. Otherwise $\theta=\varepsilon_{c}=\gamma^{2}$, contradicting that $\varepsilon_{c}$ is a fundamental unit.

If $(a, c)=1$ then $\theta=1$ and $\gamma^{2}=1$. Hence, by (1.8), we have

$$
a^{\prime}+b^{\prime} \sqrt{c}= \begin{cases}a, & \text { if } c \neq 1(\bmod 4), \\ 2 a, & \text { if } c \equiv 1(\bmod 4) .\end{cases}
$$

From Theorem 1 (examining cases) we see that $L / K$ possesses a NRIB only when

or

$$
a \equiv 1(\bmod 4)
$$

$$
c \equiv 3(\bmod 4), \quad a \equiv 3(\bmod 4), \quad t \equiv 0(\bmod 2), \quad u \equiv 1(\bmod 2) \text {. }
$$

If $(a, c) \neq 1$ then $\theta=\varepsilon_{c}$ and $\gamma^{2}=(a, c) \varepsilon_{c}$. Hence, by (1.8), (1.10) and Lemma 3, we have 


$$
a^{\prime}+b^{\prime} \sqrt{c}=\left\{\begin{array}{rr}
\frac{a}{(a, c)}(t-u \sqrt{c}), & \text { if } c \equiv 1(\bmod 4), \\
& \text { or } \\
c \equiv 5(\bmod 8), F(c)=1, & \\
\frac{2 a}{(a, c)}(t-u \sqrt{c}), & \text { if } c \equiv 5(\bmod 8), F(c)=-1 \text { or } c \equiv 1(\bmod 8) .
\end{array}\right.
$$

Again by Theorem 1, after an examination of cases, we see that $L / K$ possesses a NRIB only when

$$
c \equiv 1(\bmod 4) \text { or } c \neq \equiv 1(\bmod 4), \frac{a t}{(a, c)} \equiv 1(\bmod 4) \text {. }
$$

We note that Theorem 3 extends work of Brinkhuis [1] and Gras [2].

\section{5. $L$ pure: Proof of Theorem 4}

Let $L$ be a pure quartic field so that $L=Q(\sqrt{b \sqrt{c}})$, where $b$ and $c$ are squarefree integers with $(b, c) \neq( \pm 2,-1)$ and $c+b$ if $c \neq-1$. Set $K=Q(\sqrt{c})$. Suppose $L / K$ has a RIB and that $d(L / K)$ is squarefree. By Theorem 1 of [9] and the tables in [3] or [4] the latter assumption implies that

$$
c \equiv 7(\bmod 8), \quad b \equiv 2(\bmod 4) .
$$

The first assumption guarantees the existence of $\gamma \in O_{K}$ and $\theta \in U_{K}$ such that

$$
2(b, c)=\gamma^{2} \theta \text {. }
$$

We show that $\theta= \pm 1$ is impossible. Suppose $\theta= \pm 1$ then $a^{\prime}+b^{\prime} \sqrt{c}=$ $b \sqrt{c} / \pm 2(b, c)$ so $a^{\prime}=0$. As $L / K$ possesses a RIB, by Theorem 2 of [9], we see that $a^{\prime}$ is odd, a contradiction.

We now treat two cases according as $c<0$ or $c>0$. If $c<0$ we must have $c=-1, \theta= \pm i$. Thus $a^{\prime}=\mp b / 2 \equiv 1(\bmod 2)$ and $L / K$ has a NRIB by Theorem 1 . If $c>0$ we have without loss of generality $\theta= \pm \varepsilon_{c}$. Further $\theta=2(b, c) / \gamma^{2}>0$ so $\theta=\varepsilon_{c}$. Also $N\left(\varepsilon_{c}\right)=N(\theta)=4(b, c)^{2} / N(\gamma)^{2}>0$ so $N\left(\varepsilon_{c}\right)=1$. Hence $a^{\prime}=b c u / 2(b, c)$. As $L / K$ possesses a RIB, by Theorem 2 of [9], $a^{\prime}$ is odd, so that $u \equiv 1(\bmod 2)$, and thus $t \equiv 0(\bmod 2)$. By Theorem $1(c \equiv 3(\bmod 4)$, (iv), (vi)) $L / K$ has a NRIB.

\section{Examples}

We conclude this paper with some examples.

Example 1. We consider $L=Q(\sqrt{-17}+18 \sqrt{5})$. The quadratic subfield of $L$ is $K=Q(\sqrt{5})$. It was shown in [9, Example 2] that $L / K$ possesses a RIB. Here $a=-17, b=18, c=5, \mu=-17+18 \sqrt{5}=((-1+3 \sqrt{5}) / 2)^{3}, R=S=((-1+3 \sqrt{5})$ 
/2), $T=(1), \gamma=(-1+3 \sqrt{5}) / 2, \quad \varepsilon_{5}=(1+\sqrt{5}) / 2, \quad t=u=1, F(5)=1,\left(a^{\prime}+b^{\prime} \sqrt{c}\right) / 2=$ $\mu / \gamma^{2}=(-1+3 \sqrt{5}) / 2, a^{\prime}=-1, b^{\prime}=3$. Thus by Theorem $1 \quad(c \equiv 5(\bmod 8)$, (iii)) $L / K$ has a NRIB, which can be taken as

$$
\left\{\frac{1-\sqrt{5}}{4}+\frac{1}{2} \sqrt{\frac{-1+3 \sqrt{5}}{2}}, \frac{1-\sqrt{5}}{4}-\frac{1}{2} \sqrt{\frac{-1+3 \sqrt{5}}{2}}\right\} \text {. }
$$

Example 2. We take $L=Q(\sqrt{-5}, \sqrt{-1})$ and $K=Q(\sqrt{-5})$. Here $a=-1$, $b=0, c=-5, \mu=-1, R=S=T=O_{K}, \gamma=1, L / K$ has a RIB by [9, Theorem 2], and $d(L / K)$ is squarefree. However, $a \neq 1(\bmod 4)$ so, by Theorem $3, L / K$ does not possess a NRIB.

Example 3. Let $a$ and $b$ be integers with $(a, b)$ squarefree and $a+b v$ not a square in $K=Q(i)$. Then $L=Q(\sqrt{a+b i})$ possesses a NRIB over $K$ if and only if

$$
a \equiv 1(\bmod 2), \quad b \equiv 0(\bmod 4)
$$

or

$$
a \equiv 0(\bmod 8), \quad b \equiv 2(\bmod 4) \text {. }
$$

Example 4. Let $a$ and $b$ be integers with $(a, b)$ squarefree and $a+b \sqrt{-3}$ not a square in $K=Q(\sqrt{-3})$. Then $L=Q(\sqrt{a+b \sqrt{-3}})$ possesses a NRIB over $K$ if and only if

or

$$
a \equiv 1(\bmod 2), \quad b \equiv 0(\bmod 2), \quad a+b \equiv 1(\bmod 4)
$$

$$
a \equiv 6(\bmod 8), \quad b \equiv 2(\bmod 4), \quad a-b \equiv 0,12(\bmod 16) .
$$

Example 5. $L=Q(\sqrt{-7}, \sqrt{5})$ has a NRIB over $K=Q(\sqrt{-7})$, namely,

$$
\left\{\frac{1+\sqrt{5}}{2}, \frac{1-\sqrt{5}}{2}\right\} \text {. }
$$

Example 6. This example was considered by Kawamoto [5, Remark 12]. $L=Q(\sqrt{3+2 \sqrt{6}})$ has a RIB over $K=Q(\sqrt{6})$, namely

$$
\left\{1, \frac{1}{2}(1+\sqrt{6}+\sqrt{3+2 \sqrt{6}})\right\},
$$

but, by Theorem $1, L$ does not have a NRIB over $K$. Compare Sze [10, Theorem 1].

\section{REFERENCES}

[1] J. BRINKHUIS, Embedding problems and Galois modules, Doctoral Thesis, University of Leiden (1981). 
[2] M.-N. Gras, Bases d'entiers dans les extensions cycliques de degré 4 de $\boldsymbol{Q}$, Seminar on Number Theory, 1982-1983, Exp. No. 11, 11 pp.

[3] J.G. Huard, B.K. Spearman and K.S. Williams, Integral bases for quartic fields with quadratic subfields, Carleton University Centre for Research in Algebra and Number Theory Mathematical Research Series, No. 4, 1991, 44 pp.

[4] J.G. Huard, B.K. Spearman and K.S. Williams, Integral bases for quartic fields with quadratıc subfields, J. Number Theory, 51 (1995), 87-102.

[5] F. Kawamoto, Normal integral bases and divisor polynomials, Ph. D. Thesis, Gakushuin University (1986).

[6] R. MAssy, Formules de construction de bases normales d'entiers relatives, C.R. Acad. Sci. Paris Sér. I Math., 313 (1991), 477-482.

[7] R. MASsY, Bases normales d'entiers relatives quadratiques, J. Number Theory, 38 (1991), 216-239.

[8] B.K. Spearman and K.S. Williams, Cyclic quartic fields with relative integral bases over their quadratic subfields, Proc. Amer. Math. Soc., 103 (1988), 687-694.

[9] B.K. Spearman and K.S. Williams, Relative integral bases for quartic fields over quadratic subfields, Acta Math. Hungar., 70 (1996), 185-192.

[10] A. SzE, A normal integral basis theorem, J. Algebra, 66 (1980), 544-549.

Department of Mathematics and Statistics

Okanagan University College

Kelowna, British Columbia, Canada

V1V 1V7

e-mail : bkspearm@okanagan.bc.ca

Department of Mathematics and Statistics

Carleton University

OtTawa, Ontario, Canada

K1S 5B6

e-mail :williams@math.carleton.ca 